PHYSICAL REVIEW D 94, 123521 (2016)

\title{
Shortcomings of new parametrizations of inflation
}

\author{
Jérôme Martin \\ Institut d'Astrophysique de Paris, UMR 7095-CNRS, Université Pierre et Marie Curie, \\ 98 bis boulevard Arago, 75014 Paris, France \\ Christophe Ringeval ${ }^{\dagger}$ \\ Centre for Cosmology, Particle Physics and Phenomenology, Institute of Mathematics and Physics, \\ Louvain University, 2 Chemin du Cyclotron, 1348 Louvain-la-Neuve, Belgium \\ Vincent Vennin \\ Institute of Cosmology \& Gravitation, University of Portsmouth, Dennis Sciama Building, \\ Burnaby Road, Portsmouth PO1 3FX, United Kingdom \\ (Received 28 September 2016; published 22 December 2016)

\begin{abstract}
In the hope of avoiding model dependence of the cosmological observables, phenomenological
\end{abstract} \\ parametrizations of cosmic inflation have recently been proposed. Typically, they are expressed in terms of \\ two parameters associated with an expansion of the inflationary quantities matching the belief that inflation \\ is characterized by two numbers only, the tensor-to-scalar ratio and the scalar spectral index. We give \\ different arguments and examples showing that these new approaches are either not generic or insufficient \\ to make predictions at the accuracy level needed by the cosmological data. We conclude that disconnecting \\ inflation from high energy physics and gravity might not be the most promising way to learn about the \\ physics of the early Universe.
}

DOI: 10.1103/PhysRevD.94.123521

\section{INTRODUCTION}

With the advent of precision cosmology, it is now possible to observationally probe the early Universe and its front-runner paradigm, cosmic inflation [1-12]. When the mechanism of inflation was discovered, only a few models $[2,5,6]$, making simple predictions were proposed. However, over time, many more scenarios, often complex, were devised. This has resulted in a situation where literally hundreds of inflationary models are a priori possible. This should not come as a surprise given that, in order to build an inflationary model, one has to extrapolate high energy physics, or gravity, by many orders of magnitude, in a regime where nothing is experimentally known. In some sense, the profusion of proposed models is due to our lack of knowledge of physics beyond the electroweak scale and not to a lack of predictability of inflation.

However, with the recent release of the Planck 2013 and 2015 data [13-16], the Augean stables have started to be cleaned up. Indeed, models of inflation generating non-negligible isocurvature perturbations, large nonGaussianities and/or significant features in the power spectrum are, for the moment, disfavored by observations. Single-field slow-roll models of inflation with a minimal kinetic term therefore appear to be preferred [17-22], even

\footnotetext{
*jmartin@iap.fr

christophe.ringeval@uclouvain.be

¥vincent.vennin@port.ac.uk
}

if a large number of other scenarios still remain compatible with the data [23-26].

An alternative approach to systematic model comparison consists in considering model independent parametrizations of inflation. Such parametrizations aim at embracing all models at once while avoiding difficult questions related to specifying a potential $V(\phi)$, as for instance discussing the physical values of its parameters, possible quantum corrections or interaction of the inflaton field with other sectors. Such a proposal was first implemented within the slow-roll formalism [27-32]. It has been successfully applied to models with nonminimal kinetic terms [33-38], multifield inflation and modified gravity [39-44] while being used for non-Gaussianities [45-49] as well. Classes of inflationary models could also be devised owing to slow roll, as for instance the Schwarz and Terrero-Escalante (STE) classification [50] where only one of the three classes survived the Planck measurements [20]. If the microphysics is considered instead, the effective theory of inflation [51,52] can also be a way to parametrize deviations from the simplest physical setups.

These parametrizations yield a vast range of observable predictions, precisely because they are intended to be model independent and designed to describe many possible scenarios. However, knowing a preferred range for the tensor-to-scalar ratio $r$ would greatly help the design of future missions aiming at measuring the $B$-polarization of the cosmic microwave background (CMB) radiation. Similarly, the amount of non-Gaussianities expected within 
various classes of inflationary models is valuable information for future galactic surveys such as Euclid [53].

For these reasons and despite the existence of the slow-roll formalism, new "simple" parametrizations have recently been proposed that aim at narrowing down inflationary predictions. Moreover, it has been suggested that, at the observational level, inflation can be reduced to two numbers only (the scalar power spectrum spectral index and the tensor-to-scalar ratio), which was argued to further motivate the introduction of these new frameworks. These new parametrizations include, among others, the truncated horizon-flow formalism [54-57], the "universality classes" [58-62], and designing a simple hydrodynamical description of inflation $[63,64]$.

In this short article, we investigate whether these new approaches can be further used to constrain the physics of the early Universe (for issues with the truncated horizonflow approach, see Refs. [65-67]). The paper is organized as follows. In Sec. II, we explain why expanding observables in the so-called "large $N$ limit" ( $N$ being the number of $e$-folds) is not always consistent. We also show that the number of universality classes becomes large beyond the leading order where they thus provide a more complex classification. In Sec. III, we show that the large $N$ limit gives insufficiently accurate predictions for the spectral index $n_{\mathrm{s}}$ and the tensor-to-scalar ratio $r$. With respect to the Planck 2015 confidence intervals, these inaccuracies range from one to two sigma or more, depending on the underlying inflationary scenario. In Sec. IV, it is shown that this approach does not allow one to consistently incorporate reheating nor to derive constraints on its expansion history. Section V is dedicated to the alternative parametrization of inflation in which one specifies the equation of state parameter $w(N)$ as a function of the number of $e$-folds [64]. Such a parametrization is shown to be free of the above-mentioned issues for the simple reason that, at the background level, it ends up being equivalent to choosing a specific potential for a single scalar field. At the perturbative level, it is either incomplete because the speed of sound and the nonadiabatic pressure have to be specified (see also Ref. [68]) or implicitly equivalent to a perturbed single scalar field. In Sec. VI, we stress the fact that all these alternative approaches, independently of their internal consistencies, are not well suited to perform Bayesian statistical analysis of the cosmological data. Finally, in the conclusion, we argue that these frameworks do not allow one to connect inflation and high energy physics (modified gravity included).

\section{NOT UNIVERSAL}

\section{A. General definitions}

In the standard formulation, a single-field slow-roll model of inflation is specified by a potential $V(\phi)$. Then, the behavior of the system is completely controlled by the Friedmann-Lemaitre and Klein-Gordon equations. In general, these equations cannot be solved analytically, and one has to use either an exact numerical integration [69-75] or an approximation scheme. Given that, during inflation, the Hubble parameter $H$ is almost constant, one can define an analytical expansion in terms of small parameters that are the successive derivatives of $H$. These are called "Hubble-flow" parameters and are given by [31]

$$
\epsilon_{n+1}=\frac{\mathrm{d} \ln \left|\epsilon_{n}\right|}{\mathrm{d} N}, \quad n \geq 0,
$$

where $N=\ln \left(a / a_{\mathrm{ini}}\right)$ is the number of $e$-folds and $\epsilon_{0} \equiv H_{\text {ini }} / H$. Using these functions, one can then perturbatively calculate the power spectra of scalar and tensor modes. At leading order, the expressions of the scalar spectral index, tensor-to-scalar ratio and scalar running are given by $[29-32,37,38]$

$$
\begin{aligned}
n_{\mathrm{s}} & =1-2 \epsilon_{1 *}-\epsilon_{2 *}+\mathcal{O}\left(\epsilon^{2}\right), \\
r & =16 \epsilon_{1 *}+\mathcal{O}\left(\epsilon^{2}\right), \\
\alpha_{\mathrm{s}} & =\mathcal{O}\left(\epsilon^{2}\right) .
\end{aligned}
$$

These formulas are evaluated at the field value $\phi_{*}$ where the pivot scale at which these quantities are defined exits the Hubble radius during inflation. It is expressed in terms of $\Delta N_{*} \equiv N_{\mathrm{end}}-N_{*}$ as

$$
\Delta N_{*}=-\frac{1}{M_{\mathrm{P} 1}^{2}} \int_{\phi_{*}}^{\phi_{\text {end }}} \frac{V}{V^{\prime}} \mathrm{d} \phi,
$$

where $\phi_{\text {end }}$ satisfies $\epsilon_{1}\left(\phi_{\text {end }}\right)=1$ and denotes the value of $\phi$ at the end of inflation. Here, primes denote differentiation with respect to $\phi$.

One can also introduce "slow-roll" parameters, noted $\epsilon_{v n}$ in the following and defined directly from the inflaton potential and its derivatives, namely, [76]

$\epsilon_{v 1}=\frac{M_{\mathrm{P} 1}^{2}}{2}\left(\frac{V^{\prime}}{V}\right)^{2}, \quad \epsilon_{v 2}=2 M_{\mathrm{P} 1}^{2}\left[\left(\frac{V^{\prime}}{V}\right)^{2}-\frac{V^{\prime \prime}}{V}\right]$,

the hierarchy, as for the Hubble-flow functions, being also infinite with, for instance, $\epsilon_{v 2} \epsilon_{v 3}=-M_{\mathrm{P} 1}^{2} \epsilon_{v 2}^{\prime} V^{\prime} / V$, $\epsilon_{v 3} \epsilon_{v 4}=-M_{\mathrm{P} 1}^{2} \epsilon_{v 3}^{\prime} V^{\prime} / V$ and so on. At leading order, one can show that

$$
\epsilon_{1}=\epsilon_{v 1}, \quad \epsilon_{2}=\epsilon_{v 2} .
$$

In practice, for a given potential $V(\phi)$, one first calculates the functions $\epsilon_{v 1}(\phi)$ and $\epsilon_{v 2}(\phi)$. As just explained, this directly leads to the Hubble flow functions $\epsilon_{1}$ and $\epsilon_{2}$ through Eq. (7). The end of inflation can be determined 
from the condition $\epsilon_{1}\left(\phi_{\text {end }}\right)=\epsilon_{v 1}\left(\phi_{\text {end }}\right)=1$. Notice that, a priori, using $\epsilon_{1}=\epsilon_{v 1}$ at the end of inflation is not justified since, by definition, slow roll is violated in this regime. But, in practice, this leads to a small error that can be neglected. Finally, Eq. (5) allows us to derive $\phi_{*}=\phi\left(\Delta N_{*}\right)$, and, putting everything together, one arrives at

$$
\epsilon_{n *}=\epsilon_{v n *}=\epsilon_{v n}\left(\phi_{*}\right)=\epsilon_{v n}\left[\phi\left(\Delta N_{*}\right)\right] .
$$

As a consequence, choosing a value for $\Delta N_{*}$ (which depends on the reheating epoch and the postinflationary history of the Universe, see Sec. IV) leads to a definite value for $\epsilon_{n *}$ and, therefore, to a prediction of the model since $n_{\mathrm{s}}$ and $r$ are now explicitly known.

At the time of precision cosmology, it is in fact important to go to next-to-leading order. This is highly nontrivial since this causes new problems. For instance, the calculation of the power spectra becomes much more involved because the standard method, based on the Bessel function method, is no longer available. Fortunately, there exist other methods, for instance based on the Wentzel-Kramers-Brillouin approximation (or its extension such as the uniform approximation), which allows one to go beyond the leading order. This leads to the following expressions [30,31,37,38],

$$
\begin{aligned}
n_{\mathrm{s}}= & 1-2 \epsilon_{1 *}-\epsilon_{2 *}-(3+2 C) \epsilon_{1 *} \epsilon_{2 *} \\
& -2 \epsilon_{1 *}^{2}-C \epsilon_{2 *} \epsilon_{3 *}+\mathcal{O}\left(\epsilon^{3}\right), \\
r= & 16 \epsilon_{1 *}\left(1+C \epsilon_{2 *}\right)+\mathcal{O}\left(\epsilon^{3}\right), \\
\alpha_{\mathrm{s}}= & -2 \epsilon_{1 *} \epsilon_{2 *}-\epsilon_{2 *} \epsilon_{3 *}+\mathcal{O}\left(\epsilon^{3}\right),
\end{aligned}
$$

where $C \equiv \gamma+\ln 2-2, \gamma$ being the Euler constant. Another modification that arises at next-to-leading order is that the functions $\epsilon_{n}$ and $\epsilon_{v n}$ no longer coincide. Indeed, from the slow-roll parameters (6) calculated by means of the potential, the Hubble-flow functions at second order are given by $[66,76]$

$$
\begin{aligned}
& \epsilon_{1}=\epsilon_{v 1}\left(1-\frac{\epsilon_{v 2}}{3}\right)+\mathcal{O}\left(\epsilon_{v}^{3}\right), \\
& \epsilon_{2}=\epsilon_{v 2}\left(1-\frac{\epsilon_{v 2}}{6}-\frac{\epsilon_{v 3}}{3}\right)+\mathcal{O}\left(\epsilon_{v}^{3}\right), \\
& \epsilon_{3}=\epsilon_{v 3}\left(1-\frac{\epsilon_{v 2}}{3}-\frac{\epsilon_{v 4}}{3}\right)+\mathcal{O}\left(\epsilon_{v}^{3}\right) .
\end{aligned}
$$

A priori, this also means that the determination of the end point of inflation is modified since, at this order, we no longer have $\epsilon_{1}=\epsilon_{v 1}$. However, we have already seen that the slow-roll approximation is anyway violated for $\epsilon_{1}=1$, and, therefore, adding a correction in this regime cannot be trusted. For this reason, one still uses the condition $\epsilon_{v 1}=1$, as well as Eq. (5), to determine the end of inflation and the trajectory, respectively. The error induced on $\Delta N_{*}$ ends up being small, of a few $e$-folds at most [69-75], the reason being that when slow roll is violated inflation cannot be sustained for many $e$-folds.

Following the same logics as explained before, one can finally find the function $\epsilon_{n *}=\epsilon_{n}\left(\Delta N_{*}\right)$, this time at next-to-leading order. In this way, one can obtain more accurate predictions if needed.

\section{B. One universality class}

Recently, various works have tried to parametrize inflation by a first order expansion of $\epsilon_{1 *}$ in the small number $1 / \Delta N_{*} \ll 1$. Originally, it was postulated that most interesting inflationary scenarios should lead to [59]

$$
\epsilon_{1 *}=\frac{\beta}{\left(\Delta N_{*}\right)^{\alpha}}+\cdots,
$$

the higher order terms being assumed to be negligible. The motivation in doing so is the remark that, assuming $\Delta N_{*}=\mathcal{O}\left(10^{2}\right)$, the deviations expected from scale invariance for the simplest case $\alpha=1$ are of the order $10^{-2}$, which is, up to a factor of a few, the current measurement of the spectral index $n_{\mathrm{s}}-1$.

In fact, it is easy to find models for which Eq. (13) is not true. For instance, Khäler Moduli Inflation II (KMIII),

$$
V(\phi) \propto 1-\bar{\alpha}\left(\frac{\phi}{M_{\mathrm{P} 1}}\right)^{4 / 3} \exp \left[-\bar{\beta}\left(\frac{\phi}{M_{\mathrm{P} 1}}\right)^{4 / 3}\right],
$$

where $\bar{\alpha}$ and $\bar{\beta}$ are two model parameters, one of the best models according to the Planck data (this model belongs to the "plateau inflation" category), leads to [19]

$$
\begin{aligned}
& \epsilon_{v 1 *}=\frac{\ln ^{5 / 2}\left(16 \bar{\alpha} \sqrt{\frac{9 \bar{\beta}^{1 / 2}}{8}} \Delta N_{*}\right)}{324 \bar{\beta}^{3 / 2} \Delta N_{*}^{2}}+\mathcal{O}\left(\frac{1}{\Delta N_{*}^{3}}\right), \\
& \epsilon_{v 2 *}=\frac{2}{\Delta N_{*}}+\mathcal{O}\left(\frac{1}{\Delta N_{*}^{2}}\right) .
\end{aligned}
$$

Let us stress that this model is not a contrived scenario designed to artificially produce a dependence different from the one of Eq. (13). It is a string-inspired model that fits the data very well [19] and is one example among others for which the first Hubble flow function does not scale as an inverse power law of $\Delta N_{*}$.

\section{Several classes}

The number of universality classes was then extended in Ref. [60], in which power-law terms (13) belong to the "perturbative" category, purely exponential terms are "nonperturbative" and logarithm functionals such as KMIII belong to the "logarithmic" class. This shows that in practice, to design a complete set of universality classes, one has to study all inflationary models, as done in Refs. $[19,20]$, compute their predictions and, then, attempt to organize them into universality classes. 
As a consequence, universality classes do not dispense one with a systematic study of theoretically motivated inflationary models. In this sense, they do not seem more generic than the standard approach but should rather be seen as a way to classify models, similar to the STE classification [50] for instance. In particular, if new models are proposed in the future, the introduction of additional universality classes may be necessary.

\section{Even more classes at next-to-leading order}

A leading order term of the form $\propto 1 /\left(\Delta N_{*}\right)^{\alpha}$ does not guarantee that the next-to-leading order terms (the importance of which will be demonstrated in Sec. III) are similar and the expansion simple. In this section, we show that a model can be perturbative at leading order while being logarithmic at next-to-leading order for instance. In principle, this requires introducing a new classification at next-to-leading order and further extending the number of classes that are necessary to describe all situations.

Let us consider one of the simplest and currently favored models of inflation, namely, the Starobinsky inflation model [2] (SI), for which the potential is given by

$$
V(\phi) \propto\left(1-e^{-\sqrt{2 / 3} \phi / M_{\mathrm{P} 1}}\right)^{2}
$$

Up to the overall normalization, this model has no free parameter. Jumping straight to the result, one obtains

$$
\begin{aligned}
n_{\mathrm{s}} & =1-\frac{2}{\Delta N_{*}}+\frac{1}{\left(\Delta N_{*}\right)^{2}}\left[-\frac{5}{3}+\sqrt{3}-2 C-\frac{3}{2} \ln \left(1+\frac{2}{\sqrt{3}}\right)+\frac{3}{2} \ln \left(\frac{4}{3} \Delta N_{*}\right)\right]+\mathcal{O}\left(\frac{1}{\Delta N_{*}^{3}}\right), \\
r & =\frac{12}{\left(\Delta N_{*}\right)^{2}}-\frac{2}{\left(\Delta N_{*}\right)^{3}}\left[4+6 \sqrt{3}-12 C-9 \ln \left(1+\frac{2}{\sqrt{3}}\right)+9 \ln \left(\frac{4}{3} \Delta N_{*}\right)\right]+\mathcal{O}\left(\frac{1}{\Delta N_{*}^{4}}\right), \\
\alpha_{\mathrm{s}} & =-\frac{2}{\left(\Delta N_{*}\right)^{2}}+\frac{1}{\left(\Delta N_{*}\right)^{3}}\left[-\frac{25}{6}+2 \sqrt{3}-3 \ln \left(1+\frac{2}{\sqrt{3}}\right)+3 \ln \left(\frac{4}{3} \Delta N_{*}\right)\right]+\mathcal{O}\left(\frac{1}{\Delta N_{*}^{4}}\right) .
\end{aligned}
$$

Although the first terms of the series are inverse power laws of $\Delta N_{*}$, the higher order terms are not just given by higher inverse power laws but also contain logarithms of $\Delta N_{*}$. Let us also notice that these expressions extend the ones of Ref. [59] [see Eq. (34)]. ${ }^{1}$ In general, a classification into universality classes at next-to-leading order can therefore not be done without largely increasing the number of classes.

\section{E. Validity of the expansion}

In practice, the " $1 / \Delta N_{*}$ expansion" is not an expansion in $1 / \Delta N_{*}$ alone but usually also involves the parameters of the potential. This implies that the expansion is not always valid, and, in fact, there are potentials for which it is never valid. To illustrate this statement, let us consider the small field inflation $\mathrm{SFI}_{2}$ potential

${ }^{1}$ It is interesting to explain how this was obtained. Defining $x=\phi / M_{\mathrm{P} 1}$, at leading order, the SI slow-roll trajectory reads

$$
x_{*}=\sqrt{\frac{3}{2}}\left[-f_{*}-\mathrm{W}_{-1}\left(-e^{-f_{*}}\right)\right],
$$

where $\mathrm{W}_{-1}$ is the "-1" branch of the Lambert function and

$$
f_{*} \equiv \frac{4}{3} \Delta N_{*}-\sqrt{\frac{2}{3}} x_{\text {end }}+e^{\sqrt{2 / 3} x_{\text {end }}}
$$

with $x_{\text {end }} \equiv \sqrt{3 / 2} \ln (1+2 / \sqrt{3})$. Plugging this trajectory into Eq. (6), and expanding it at next-to-leading order, one obtains

$$
\epsilon_{v 1 *}=\frac{3}{4 \Delta N_{*}^{2}}-\frac{9}{8 \Delta N_{*}^{3}}\left[\frac{2}{\sqrt{3}}-\ln \left(1+\frac{2}{\sqrt{3}}\right)+\ln \left(\frac{4}{3} \Delta N_{*}\right)\right]+\mathcal{O}\left(\frac{1}{\Delta N_{*}^{4}}\right) .
$$

The formula for $r$ found in Ref. [59], namely,

$$
r_{[59]}=\frac{12}{\Delta N_{*}^{2}}-\frac{18}{\Delta N_{*}^{3}} \ln \left(\Delta N_{*}\right),
$$

corresponds to taking the expression (20), and using it in Eq. (3). Eq. (21) is valid at order $\ln \left(\Delta N_{*}\right) / \Delta N_{*}^{3}$ but not at next-to-leading order in slow roll where higher $1 / \Delta N_{*}^{3}$ terms appear. In particular, this amounts to ignoring the numerical factor $2 / \sqrt{3}-\ln (1+2 / \sqrt{3})+\ln (4 / 3) \simeq 0.67$, which is in fact not completely negligible compared to $\ln \Delta N_{*}\left(\simeq 3.7\right.$ for $\left.\Delta N_{*} \simeq 40\right)$. 


$$
V(\phi) \propto 1-\left(\frac{\phi}{\mu}\right)^{2},
$$

which has one free parameter $\mu$. Making use of the techniques introduced in Sec. II A and defining $x \equiv \phi / \mu$, the slow-roll trajectory reads

$$
x_{*}=\sqrt{-\mathrm{W}_{0}\left(-e^{-f_{*}}\right)},
$$

where $\mathrm{W}_{0}$ denotes the " 0 " branch of the Lambert function and $f_{*}$ is given by

$$
f_{*} \equiv 4 \Delta N_{*} \frac{M_{\mathrm{P} 1}^{2}}{\mu^{2}}+x_{\text {end }}^{2}(\mu)-2 \ln \left[x_{\text {end }}(\mu)\right]
$$

In this equation, $x_{\text {end }}$ is the value of $x$ at the end of inflation,

$$
x_{\mathrm{end}}=\frac{1}{\sqrt{2}} \frac{M_{\mathrm{P} 1}}{\mu}\left(-1+\sqrt{1+\frac{2 \mu^{2}}{M_{\mathrm{P} 1}^{2}}}\right) .
$$

In Eq. (24), $\Delta N_{*}$ appears multiplied by the dimensionless parameter $M_{\mathrm{P} 1}^{2} / \mu^{2}$ such that performing an expansion in $1 / \Delta N_{*}$ requires some assumptions on $\mu / M_{\mathrm{P} 1}$. This shows that, as mentioned above, the small parameter of the expansion is usually not $1 / \Delta N_{*}$ alone. In the validity domain of the large $f_{*}$ limit, the Lambert function can be Taylor expanded according to $\mathrm{W}_{0}(x) \sim x$, and one obtains

$$
\begin{aligned}
\epsilon_{v 1 *}= & \frac{M_{\mathrm{P} 1}^{4}}{\mu^{4}}\left(\sqrt{1+2 \frac{\mu^{2}}{M_{\mathrm{P} 1}^{2}}}-1\right)^{2} \\
& \times \exp \left[-\frac{M_{\mathrm{P} 1}^{2}}{\mu^{2}}\left(4 \Delta N_{*}+1+\frac{\mu^{2}}{M_{\mathrm{P} 1}^{2}}-\sqrt{1+2 \frac{\mu^{2}}{M_{\mathrm{P} 1}^{2}}}\right)\right] \\
& +\mathcal{O}\left(e^{-8 \frac{M_{\mathrm{P} 1}^{2}}{\mu^{2}} \Delta N_{*}}\right), \\
\epsilon_{v 2 *}= & 4 \frac{M_{\mathrm{P} 1}^{2}}{\mu^{2}}+\mathcal{O}\left(e^{-4 \frac{M_{\mathrm{P} 1}^{2}}{\mu^{2}} \Delta N_{*}}\right) .
\end{aligned}
$$

Let us notice that $\epsilon_{1 *}$ does not behave as in Eq. (13) but belongs to the nonperturbative class of Ref. [60].

This expansion is valid as soon as $f_{*} \gg 1$, which is the case if $\mu \ll M_{\mathrm{P} 1}$. This limit is, however, inconsistent with the slow-roll approximation because $\epsilon_{v 2 *} \gg 1$. Let us stress that, as soon as slow roll is violated, one can no longer make use of Eqs. (2) and (3) to derive analytical expressions for the spectral index and the tensor-to-scalar ratio in terms of the $\epsilon_{n *}$ and, thus, in terms of $\Delta N_{*}$. From Eq. (27), one has $\epsilon_{v 2 *}<1$ for $\mu>2 M_{\mathrm{P} 1}$. As a result, Eq. (24) shows that $f_{*}$ could be made reasonably large in the large $\Delta N_{*}$ limit and provided $\mu /\left(2 M_{\mathrm{P} 1}\right)$ is of order unity. Only in this very contrived situation, Eqs. (26) and (27) might be used.
We conclude that, in general, a $1 / \Delta N_{*}$ expansion cannot be performed for arbitrary values of the free parameters of the model. In this sense, it is not universal.

\section{INSUFFICIENTLY ACCURATE}

In this section, we investigate whether the $1 / \Delta N_{*}$ expansion of the Hubble-flow parameters is sufficient to match the accuracy of the present and future data. We choose to exemplify the question with two models. One is SI, the Starobinsky model [2] already introduced in Sec. II D. The other one is the small field model $\mathrm{SFI}_{4}$, with $V(\phi) \propto 1-(\phi / \mu)^{4}$, which has one free parameter $\mu$. Both models are compatible with the current data. Moreover, there are values of $\mu$ for which these two models could a priori be confused, and their disambiguation is a relevant question for future $\mathrm{CMB}$ experiments. The expressions of $n_{\mathrm{S}}$ and $r$ for SI at leading order in slow roll have already been established in the last section, namely,

$$
\begin{gathered}
n_{\mathrm{s}}=1-\frac{2}{\Delta N_{*}}+\mathcal{O}\left(\frac{\ln \Delta N_{*}}{\Delta N_{*}^{2}}\right), \\
r=\frac{12}{\Delta N_{*}^{2}}+\mathcal{O}\left(\frac{\ln \Delta N_{*}}{\Delta N_{*}^{2}}\right) .
\end{gathered}
$$

For $\mathrm{SFI}_{4}$, the field trajectory can be solved in terms of $x_{*} \equiv \phi_{*} / \mu$ as

$$
x_{*}=\sqrt{\frac{f_{*}-\sqrt{f_{*}^{2}-4}}{2}},
$$

where $f_{*}$ is defined by

$$
f_{*}=8 \Delta N_{*} \frac{M_{\mathrm{P} 1}^{2}}{\mu^{2}}+x_{\mathrm{end}}^{2}(\mu)+\frac{1}{x_{\mathrm{end}}^{2}(\mu)} .
$$

In the large $\Delta N_{*} M_{\mathrm{P} 1}^{2} / \mu^{2}$ limit, one has $x_{*} \simeq f_{*} / \sqrt{2} \simeq$ $8 \Delta N_{*} M_{\mathrm{P} 1}^{2} /\left(\sqrt{2} \mu^{2}\right)$, which gives rise to

$$
\begin{aligned}
n_{\mathrm{s}} & =1-\frac{3}{\Delta N_{*}}+\mathcal{O}\left(\frac{1}{\Delta N_{*}^{2}}\right), \\
r & =\frac{\mu^{4}}{4 M_{\mathrm{P} 1}^{4} \Delta N_{*}{ }^{3}}+\mathcal{O}\left(\frac{1}{\Delta N_{*}^{4}}\right) .
\end{aligned}
$$

As opposed to $\mathrm{SFI}_{2}$ discussed in Sec. II E, one can check that there is no slow-roll violation for any reasonable values of $\mu$ and the expansion is under control.

In Fig. 1, for various values of $\Delta N_{*}$ (represented in the color bar), we have plotted as diamonds the leading order expressions of $n_{\mathrm{s}}$ and $r$ for both SI, Eq. (29), and $\mathrm{SFI}_{4}$, Eq. (33), together with the nonapproximated slow-roll predictions (circles). For a given value of $\Delta N_{*}$, the $1 / \Delta N_{*}$ expansion significantly deviates from the nonapproximated result. For Starobinsky inflation (see the 

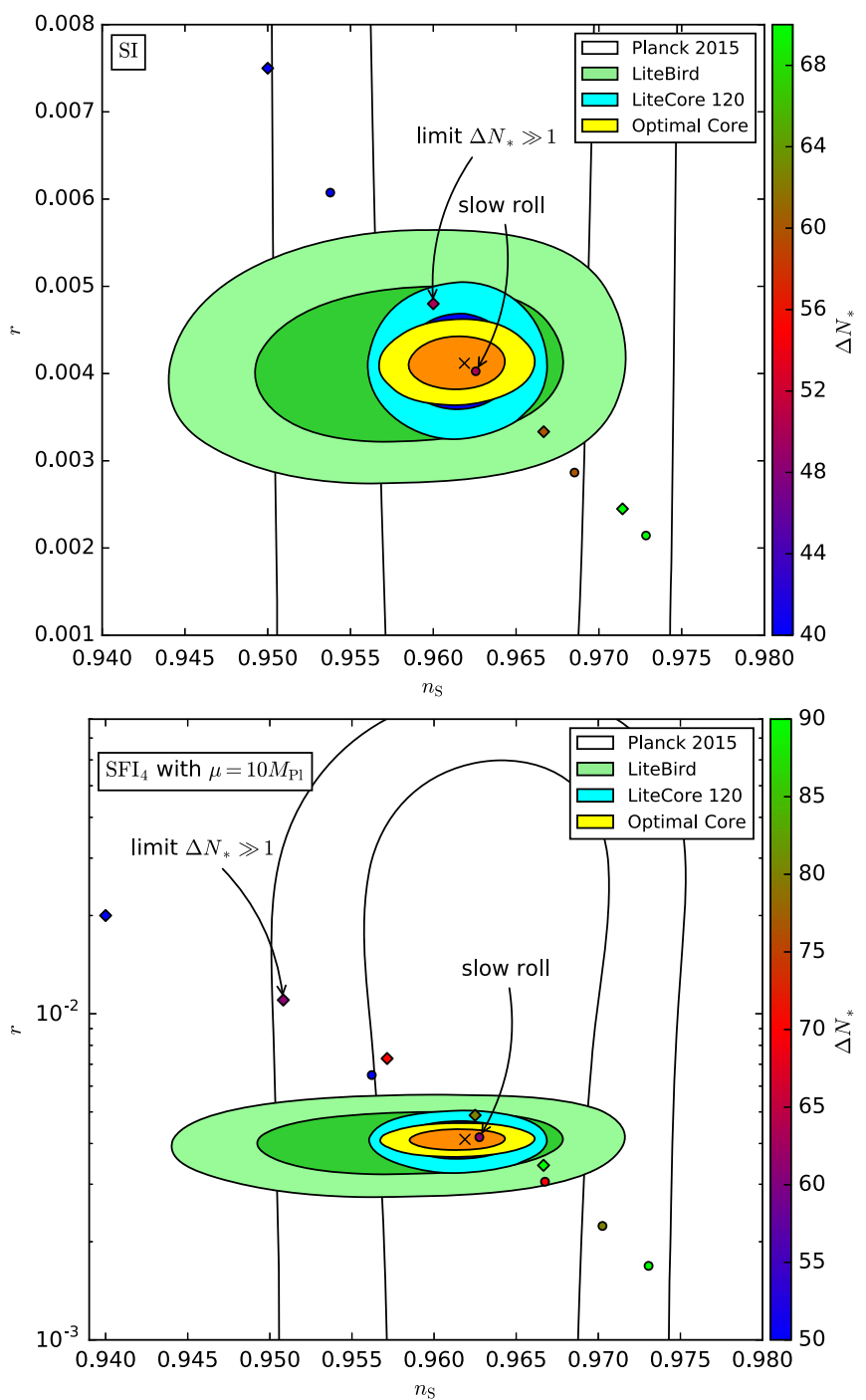

FIG. 1. Slow-roll predictions (circles) vs $1 / \Delta N_{*}$ expansions (diamonds) in the plane $\left(n_{\mathrm{s}}, r\right)$ for various values of $\Delta N_{*}$ (color bar). The upper panel shows the expected values for Starobinsky inflation (SI), while the bottom panel is for small field inflation $\mathrm{SFI}_{4}$ with $\mu=10 M_{\mathrm{P} 1}$. In both frames, we have represented the current one- and two-sigma confidence intervals from the Planck 2015 data together with the forecasts of some future experiments such as LiteBird and LiteCore (the fiducial model is denoted with the black cross). For a fixed value of $\Delta N_{*}$, the $1 / \Delta N_{*}$ expansion is not sufficiently accurate (the arrows point to the case $\Delta N_{*}=50$ for SI and $\Delta N_{*}=60$ for $\left.\mathrm{SFI}_{4}\right)$.

upper panel), the expansion in $1 / \Delta N_{*}$ yields an inaccuracy of about half to one sigma compared to the Planck 2015 constraints in the plane $\left(n_{\mathrm{s}}, r\right)$. This is barely enough for assessing the viability of the model with the Planck data. In the same figure, we have represented the expected constraints of some future CMB experiments, assuming $\mathrm{SI}$ as a fiducial. They are LiteBird [77] and various possible designs of the LiteCore mission [78]. For "Optimal Core," the case perfectly compatible with the data would appear disfavored by more than three sigmas if one would trust the result of the $1 / \Delta N_{*}$ expansion. The bottom panel of Fig. 1 shows $\mathrm{SFI}_{4}$ with $\mu=10 M_{\mathrm{P} 1}$. For such a value of $\mu$, and $\Delta N_{*} \simeq 60, \mathrm{SFI}_{4}$ matches SI. However, if one uses the $1 / \Delta N_{*}$ expansion, the resulting values of $n_{\mathrm{s}}$ and $r$ are two-sigma away from the correct values already with the Planck 2015 data, and completely off with an experiment like LiteCore.

Figure 1 suggests that the main source of error in the plane $\left(n_{\mathrm{s}}, r\right)$ is a shift in the value of $\Delta N_{*}$. For $\mathrm{SFI}_{4}$, one would need to subtract typically 20 to $\Delta N_{*}$ to recover an acceptable result. Let us notice that the uncertainties associated with slow-roll violations toward the end of inflation may also induce a discrepancy on $\Delta N_{*}$, but not more than \pm 1 , which is negligible compared to the effect discussed here. The issue comes from the expansion itself and the underlying assumption of considering the large $\Delta N_{*}$ limit.

Let us also mention that we have been fair in choosing the models displayed in Fig. 1, since other models exhibit much larger departures (as for instance $\mathrm{SFI}_{4}$ with a larger value of $\mu$ ).

\section{INCOMPATIBLE WITH REHEATING}

Expanding the Hubble-flow parameters in terms of $1 / \Delta N_{*}$ raises the question of specifying the value of $\Delta N_{*}$. The standard lore is to take the values in the range $\Delta N_{*} \in[50,60]$, or $[40,70]$, or simply postulate a fixed number like $\Delta N_{*}=60$. These values may indeed be reasonable but under various conditions.

One has to make some assumptions on how the reheating era proceeded, and on the energy scale at the end of inflation. Within a given inflationary scenario, in which the potential is specified, the energy scale at which inflation ends is fixed by the model parameters, and this is how the above-quoted numbers can actually be obtained. But this is no longer the case when one is only interested in expanding quantities around the pivot scale, as this is done in any of the $1 / \Delta N_{*}$ expansions. For instance, there are inflationary models without scalar fields in which the Hubble parameter grows during inflation [79] and for which typical values of $\Delta N_{*}$ could be completely different than [50,60]. Even for single-field inflation, depending on how reheating proceeds, Ref. [80] has shown that $\Delta N_{*}=100$ is possible. The use of an expansion in $1 / \Delta N_{*}$ is therefore questionable if one cannot predict the value of $\Delta N_{*}$, and it is easy to check in Fig. 1 the consequences of taking $\Delta N_{*}=40$ or $\Delta N_{*}=100$ on the predicted values of $n_{\mathrm{s}}$ and $r$.

The solution to this issue is to specify the inflationary potential. In this case, $\Delta N_{*}$ is given by [81-83]

$$
\begin{aligned}
\Delta N_{*}= & \frac{1-3 \bar{w}_{\text {reh }}}{12\left(1+\bar{w}_{\text {reh }}\right)} \ln \left(\frac{\rho_{\text {reh }}}{\rho_{\text {end }}}\right)-N_{0} \\
& -\frac{1}{4} \ln \left[\frac{3}{\epsilon_{1 *}} \frac{3-\epsilon_{1 *}}{3-\epsilon_{\text {lend }}} \frac{V_{\text {end }}}{V_{*}}\right]+\frac{1}{4} \ln \left(\frac{H_{*}^{2}}{M_{\mathrm{P} 1}^{2} \epsilon_{1 *}}\right),
\end{aligned}
$$


where $N_{0} \equiv \ln \left[\left(k_{*} / a_{0}\right) \tilde{\rho}_{\gamma}\right], k_{*}$ being the pivot scale and $\tilde{\rho}_{\gamma}=\mathcal{Q}_{\text {reh }} \rho_{\gamma}$ with $\rho_{\gamma}$ the total energy density stored in radiation today and $\mathcal{Q}_{\text {reh }}$ a measure of the change of relativistic degrees of freedom between the reheating epoch and today. Of course, $V$ denotes the inflationary potential, and $H_{*}^{2} / \epsilon_{1 *}=8 \pi^{2} M_{\mathrm{P} 1}^{2} P_{*}+\mathcal{O}\left(\epsilon_{1 *}\right)$ where $P_{*}$ is the amplitude of the scalar power spectrum at the pivot scale. The quantity $\rho_{\text {end }}$ denotes the energy density at the end of inflation (and depends on the model of inflation), while $\rho_{\text {reh }}$ is the energy density at the end of reheating. Finally, $\bar{w}_{\text {reh }}$ is the mean equation of state during reheating. Equation (34) shows that once the inflationary model and the parameters describing reheating are chosen (as well as the postinflationary cosmic evolution), $\Delta N_{*}$ is fully determined. In practice, however, if the inflationary Lagrangian does not specify the couplings between the inflaton and other sectors, the reheating parameters are only bounded to vary within specific ranges: $\rho_{\text {reh }}$ must be larger than the energy density at big bang nucleosynthesis (BBN) and smaller than $\rho_{\text {end }}$ while $-1 / 3<\bar{w}_{\text {reh }}<1$. This means that there is a completely determined prior range in which $\Delta N_{*}$ can vary. Postulating fixed values for $\Delta N_{*}$ misses this fact and can lead to incorrect results.

For instance, let us consider the same small field inflationary model as in Sec. III, namely, $\mathrm{SFI}_{4}$, where we fix $\mu=10 M_{\mathrm{P} 1}$. Moreover, let us assume that reheating has a mean equation of state given by $\bar{w}_{\text {reh }}=-0.3$, which could, for instance, signal a low decay rate of the inflaton or the persistence of some vacuum energy during reheating. An analysis based on Eq. (34) shows that, for this model, $\Delta N_{*} \in[18.7,55.8]$, the lower bound being obtained for a reheating at $\operatorname{BBN}\left(\rho_{\text {nuc }}^{1 / 4}=10 \mathrm{MeV}\right)$, while the upper bound corresponds to an instantaneous (or radiationlike) reheating. Within slow roll, one obtains that the spectral index $n_{\mathrm{s}} \in[0.904,0.960]$, showing that for this scenario to be within the two-sigma confidence intervals of the Planck data, reheating should be almost instantaneous (see Fig. 1).

Within the $1 / \Delta N_{*}$ expansion formalism, assuming $\Delta N_{*} \in[40,70]$ would therefore miss most of the physical range of values while encompassing all the unphysical ones $\Delta N_{*}>55.8$. For these, the reheating would end at an energy scale higher than the energy at the end of inflation. Let us stress that this issue has nothing to do with the inaccuracy of the expansion discussed in Sec. III and simply comes from the fact that one cannot arbitrarily choose a fixed number for $\Delta N_{*}$. Nevertheless, one should notice that the inaccuracy of the expansion makes the problem even worse. As can be checked in Fig. 1, if one uses the $1 / \Delta N_{*}$ expansion and tries to infer the "right value" of $\Delta N_{*}$ to make $\mathrm{SFI}_{4}$ compatible with the Planck data, one would obtain $\left.\Delta N_{*}\right|_{n_{\mathrm{s}}=0.96} \simeq 75$.

An incorrect argument against the above discussion would be to postulate that nothing can be said about the reheating era. As shown in Refs. [82,84-87], the recently released Planck data already allow us to infer some reheating physics from $\mathrm{CMB}$ data. As a result, and even if the above-mentioned limitations of the $1 / \Delta N_{*}$ expansion could be alleviated, one would still miss the opportunity to constrain reheating.

As an illustration of what the future CMB measurements could tell us about reheating, we have plotted in Fig. 2 the marginalized posterior distribution of the reheating temperature that can be inferred by the Optimal Core satellite design (solid curve). Here, the fiducial reheating history has been assumed to be with a vanishing equation of state $\bar{w}_{\text {reh }}=0$ and $T_{\text {reh }}=10^{8} \mathrm{GeV}$, a low value typical of the reheating after Starobinsky inflation [88]. Not considering the reheating effects would simply prevent us from making such a measurement.

The dashed curve shows the posterior that would be obtained by using the $1 / \Delta N_{*}$ expansion on $n_{\mathrm{s}}$ and $r$ for extracting the reheating temperature with Eq. (34). Let us notice that it would not make much sense to do so as one would still need the field potential in this equation. In any case, the inferred value of $T_{\text {reh }}$ derived in such a way is off by more than five sigmas and would wrongly prefer higher reheating temperatures, which are typical of Higgs inflation [89-92]. As such, using the $1 / \Delta N_{*}$ predictions, one would wrongly conclude that inflation is more likely to be Higgs rather than Starobinsky.

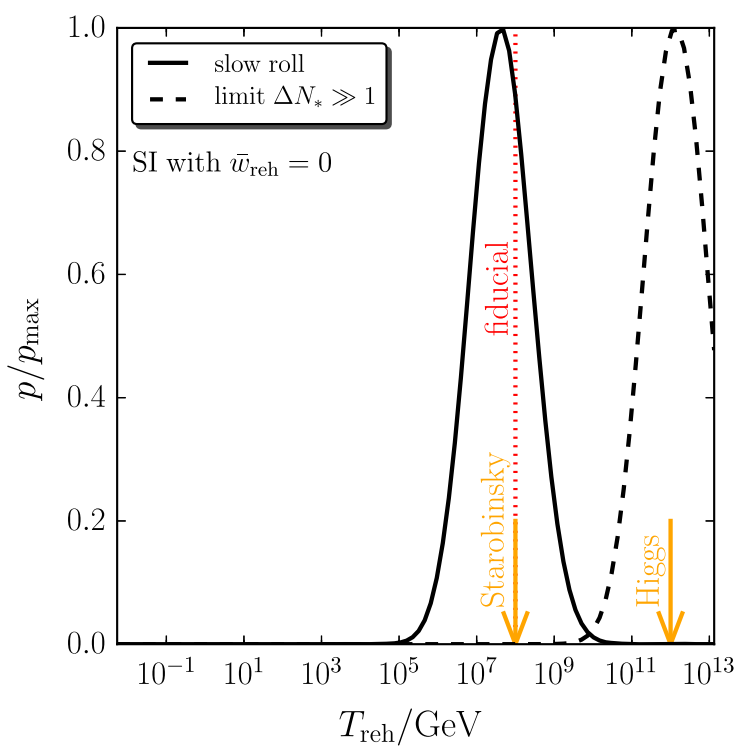

FIG. 2. Forecast of the marginalized posterior probability distribution for the reheating temperature $T_{\text {reh }}$ expected by a CMB satellite design such as "Optimal Core" (solid curve). The fiducial inflationary model is Starobinsky inflation with $\bar{w}_{\text {reh }}=0$ and $T_{\text {reh }}=10^{8} \mathrm{GeV}$. The reheating temperature can be accurately inferred. The dashed curve shows what would be obtained by using the $1 / \Delta N_{*}$ predictions. The preferred value of $T_{\text {reh }}$ is off by more than five sigmas and would favor a reheating scenario typical of Higgs inflation rather than Starobinsky inflation, an unfortunate conclusion indeed. 
Reheating is therefore a crucial part of the inflationary scenario that is now observationally constrained [93], but which cannot be reconstructed with phenomenological expansions.

\section{EQUATION-OF-STATE INFLATION?}

All of the previously discussed problems of the $1 / \Delta N_{*}$ expansion can be alleviated by simply not performing an expansion at all. Instead, following Ref. [64], one may decide to parametrize the inflationary background by specifying the evolution of the equation of state of the Universe $w(N)=P / \rho$ with respect to the number of $e$-folds $N$. This approach was already employed in Refs. $[94,95]$ and extended in Refs. [96,97] by postulating the evolution of the scale factor $a(t)$ with respect to cosmic time. As shown in these references, the functional forms chosen for $w(t)$ and $a(t)$ are equivalent to specifying the inflationary potentials of the so-called "Intermediate" (II) and "Logamediate" (LMI) models; see Secs. 5.2 and 5.4 of Ref. [98]. Here as well, we show that the choice of $w(N)$ made in Ref. [64] is equivalent to choosing a two-parameter potential that we derive.

\section{A. Background evolution}

The hydrodynamical Friedmann-Lemaître equations read

$$
H^{2}=\frac{\rho(N)}{3 M_{\mathrm{P} 1}^{2}}, \quad \frac{\mathrm{d} H}{\mathrm{~d} N}=-\frac{3}{2}[1+w(N)] H .
$$

As a result, specifying $w(N)$ fixes almost all of the nonperturbed quantities, up to the integration constant of Eq. (35); i.e. the energy scale of inflation remains, within this representation, a fundamentally unpredictable quantity. This is the first drawback of the hydrodynamical approach. When one specifies an inflationary potential, the energy scale is fixed by the overall multiplicative constant, usually referred to as $M^{4}$. For most of the inflationary models proposed so far, this parameter is usually not predicted by the theory and chosen to match the amplitude of the CMB anisotropies. In that situation, specifying $w(N)$ is indeed not worse than letting $M^{4}$ be a free parameter. However, there are inflationary models for which $M^{4}$ is predicted. For instance, this is the case for the very first models of inflation such as SI [2], Higgs inflation [89], the original ColemanWeinberg model $(\mathrm{CWI})[3,4]$ (ruled out for this very reason [99-101]), open string tachyon inflation (OSTI) [102-105] (also ruled out for this reason [106]) and dual inflation (DI) $[107,108]$. Compared to these, an inflationary background evolution given by $w(N)$ remains less predictive.

More interestingly, one can rewrite the FriedmannLemaître equations in terms of the first Hubble-flow function $\epsilon_{1}$. From its definition, one gets

$$
\epsilon_{1}(N) \equiv-\frac{\mathrm{d} \ln H}{\mathrm{~d} N}=\frac{3}{2}[1+w(N)]
$$

As a result, specifying the equation of state is strictly equivalent to postulating the evolution of the first Hubbleflow function $\epsilon_{1}(N)$. The complete Hubble-flow hierarchy $\epsilon_{n}(N)$ is then exactly known. For instance, the second and third Hubble-flow functions read

$$
\begin{aligned}
\epsilon_{2}(N) & =\frac{\dot{w}(N)}{1+w(N)}, \\
\epsilon_{3}(N) & =\frac{\ddot{w}(N)}{\dot{w}(N)}-\frac{\dot{w}(N)}{1+w(N)},
\end{aligned}
$$

where a dot denotes here the derivative with respect to $N$. Equation (36) also determines $N_{\text {end }}$, the $e$-fold at which inflation ends, given by solving $\epsilon_{1}\left(N_{\text {end }}\right)=1$. As a result, $\Delta N_{*}=N_{\text {end }}-N_{*}$ is well defined, and, up to the unknown integration constant of Eq. (35), the energy scale at which inflation ends can be uniquely determined. In particular, this allows the reheating era to be consistently considered and $\Delta N_{*}$ to be determined. At this point, one may wonder what the difference is, at the background level, compared to the more usual situation in which one specifies the field potential. The answer is none.

Indeed, comparing the Friedmann-Lemaitre equations obtained from a minimally coupled scalar field to the hydrodynamical ones [19], one gets

$$
\begin{aligned}
\left(\frac{\mathrm{d} \phi}{\mathrm{d} N}\right)^{2} & =2 M_{\mathrm{P} 1}^{2} \epsilon_{1}(N), \\
\frac{\mathrm{d} \ln V(\phi)}{\mathrm{d} N} & =-2 \epsilon_{1}(N)+\frac{\mathrm{d} \ln \left[3-\epsilon_{1}(N)\right]}{\mathrm{d} N} .
\end{aligned}
$$

Using Eq. (36), these equations can be formally integrated as

$$
\begin{aligned}
& \phi(N)=\phi_{0} \pm \sqrt{3} M_{\mathrm{P} 1} \int_{N_{0}}^{N} \sqrt{1+w(n)} \mathrm{d} n, \\
& V(N)=V_{0} \frac{1-w(N)}{1-w\left(N_{0}\right)} \exp \left\{-3 \int_{N_{0}}^{N}[1+w(n)] \mathrm{d} n\right\},
\end{aligned}
$$

where $V_{0}$ is the expected integration constant associated with energy conservation. The other integration constant, $\phi_{0}$, has no observable effect and comes from the shift symmetry of Eq. (39), while $w\left(N_{0}\right)$ can be absorbed in $V_{0}$. Eq. (40) gives a parametric representation of the field trajectory and its potential. Solving for $\phi(N)$, one then infers $N(\phi)$ which leads to $V(\phi)=V[N(\phi)]$.

As an illustration, let us recover the exact field potential associated with

$$
1+w \equiv \frac{\beta}{\left(c+\Delta N_{*}\right)^{\alpha}},
$$


where $\alpha$ and $\beta$ are two free parameters and, following Ref. [64], $c$ is a regularizing constant to avoid any divergences at the end of inflation. Let us stress that the above equation is a definition and not an expansion as in Eq. (13). In order to consistently implement the end of inflation $\epsilon_{1}\left(N_{\text {end }}\right)=1$ [or, equivalently, $w\left(N_{\text {end }}\right)=-1 / 3$ ], one has to fix $c=(3 \beta / 2)^{1 / \alpha}$. Integrating Eq. (40) and fixing $\phi_{0}=\mp \sqrt{3 \beta} /(1-\alpha / 2)$ gives the potential (some approximations of which are obtained in Ref. [64])

$$
\begin{aligned}
V(\phi)= & M^{4}\left[1-\frac{\beta}{2\left(1+\frac{2-\alpha}{2 \sqrt{3 \beta}} \frac{\phi}{M_{\mathrm{P} 1}}\right)^{\frac{2 \alpha}{2-\alpha}}}\right] \\
& \times \exp \left\{\frac{3 \beta}{1-\alpha}\left[\left(1+\frac{2-\alpha}{2 \sqrt{3 \beta}} \frac{\phi}{M_{\mathrm{P} 1}}\right)^{\frac{2(1-\alpha)}{2-\alpha}}-1\right]\right\} .
\end{aligned}
$$

In the limit $\alpha \rightarrow 1$, it is interesting to notice that this is nothing but the potential of intermediate inflation; see Sec. 5.2 of Ref. [98]. We also recover explicitly the result of Ref. [64]: for $\alpha<1$, the potential has an exponential shape reminiscent of power law inflation and LMI (although LMI is defined with a relation among the coefficients characterizing the potential which is not obtained in the present case), for $1<\alpha \leq 2$ it is of the plateau kind, and for $\alpha>2$ it is similar to small field inflation $\left[\mathrm{SFI}_{p}\right.$ with $p=2 \alpha /(\alpha-2)]$.

We conclude that, for the background evolution, choosing a function $w(N)$, or equivalently $\epsilon_{1}(N)$, is not a generic procedure but just singles out a particular $V(\phi)$, namely, a particular model of inflation. The only difference with respect to the traditional approach is that the energy scale of inflation can no longer be predicted.

\section{B. Cosmological perturbations}

Specifying $w(N)$ instead of $V(\phi)$ is, however, not enough to uniquely determine the behavior of the cosmological perturbations during inflation [68,109]. Indeed, if $\Phi_{\mathrm{B}}$ represents (the Fourier transform of) the Bardeen potential and if the Universe is dominated by a perfect fluid, then one has

$$
\begin{aligned}
\Phi_{\mathrm{B}}^{\prime \prime} & +3 \mathcal{H}\left(1+c_{\mathrm{S}}^{2}\right) \Phi_{\mathrm{B}}^{\prime}+\left[2 \mathcal{H}^{\prime}+\mathcal{H}^{2}\left(1+3 c_{\mathrm{S}}^{2}\right)\right] \Phi_{\mathrm{B}} \\
& +c_{\mathrm{S}}^{2} k^{2} \Phi_{\mathrm{B}}=\frac{a^{2}}{2 M_{\mathrm{P} 1}^{2}} \delta P_{\mathrm{nad}},
\end{aligned}
$$

where a prime denotes a derivative with respect to conformal time and $\mathcal{H}=a H$ is the conformal Hubble parameter. In this expression, $c_{\mathrm{S}}^{2} \equiv P^{\prime} / \rho^{\prime}$ is the sound speed, and $\delta P_{\text {nad }} \equiv \delta P-c_{\mathrm{S}}^{2} \delta \rho$ is the nonadiabatic pressure perturbation. If one wants the hydrodynamical perturbations to evolve as the perturbations stemming from a scalar field, the sound speed must verify the relation

$$
c_{\mathrm{S}}^{2}=1-\frac{4}{9\left[1-w(N)^{2}\right]}\left\{3+3 w(N)-\frac{\mathrm{d} \ln [1-w(N)]}{\mathrm{d} N}\right\},
$$

while the fluid must possess a nonadiabatic pressure such that

$$
\delta P_{\mathrm{nad}}=-2 M_{\mathrm{P} 1}^{2}\left(1-c_{\mathrm{S}}^{2}\right) \frac{k^{2}}{a^{2}} \Phi_{\mathrm{B}}
$$

From Eqs. (36) and (39), one indeed recovers the speed of sound associated with a perturbed scalar field

$$
c_{\mathrm{S}}^{2}=1+\frac{2 a^{2} V_{, \phi}}{3 \mathcal{H} \phi^{\prime}} .
$$

Inserting Eqs. (44) and (45) in Eq. (43) leads to an equation for the Bardeen potential which is exactly that obtained under the assumption that the dominant fluid in the Universe is a scalar field [110].

As a result, and as opposed to a scalar field, it is not sufficient to specify the background, namely, the function $w(N)$, to fix the evolution of the perturbed quantities. One should also specify the functional form of $c_{\mathrm{S}}^{2}(N)$ and $\delta P_{\text {nad }}(N)$ to have well-defined equations of motion. Conversely, implicitly assuming that the hydrodynamical perturbations evolve as the ones generated during singlefield inflation, one must have a very contrived sound speed $c_{\mathrm{S}}^{2}(N)$ and nonadiabatic pressure $\delta P_{\text {nad }}(N)$. It is hard to understand how this could be achieved without the knowledge of Eqs. (44) and (45), namely, without knowing that the underlying model is, as a matter of fact, a scalar field.

\section{STATISTICALLY FLAWED}

Finally, let us discuss whether phenomenological parametrizations of inflation are well suited to carry out statistical model comparison.

We consider a model of inflation characterized by the parameters $\boldsymbol{\theta}_{\text {inf }}$ [including the mass scale $M$ of the potential and any other parameters needed to completely specify the shape of $V(\phi)$ such as $\mu$ for SFI] and $\boldsymbol{\theta}_{\text {reh }}$ (the reheating parameters; see Sec. IV). In the slow-roll approximation, the power spectra of tensor and scalar perturbations are functions of the Hubble-flow parameters $\epsilon_{n *}$ only, which, in turn, are functions of the $\boldsymbol{\theta}_{\text {inf }}$ and $\boldsymbol{\theta}_{\text {reh }}$ parameters. As a consequence, the predictions of a model in terms of the primordial power spectra are expressed with $\mathcal{P}_{\zeta}\left(\boldsymbol{\theta}_{\text {inf }}, \boldsymbol{\theta}_{\text {reh }}\right)$ and $\mathcal{P}_{h}\left(\boldsymbol{\theta}_{\text {inf }}, \boldsymbol{\theta}_{\text {reh }}\right)$. In this manner, the slow-roll approximation is a proxy to facilitate the derivation of the power spectra as a functional of the underlying theory parameters, exactly as one would obtain from an exact integration of the inflationary perturbations [75,111]. This is a crucial difference between slow roll and the previously discussed 
alternatives which discard any underlying theoretical model.

However, in order to estimate the statistical ability of a hypothesis to explain the observed data [20,21,111-114], one must first specify the prior distributions of the underlying parameters. For the inflationary models, they are the $\boldsymbol{\theta}_{\text {inf }}$ and $\boldsymbol{\theta}_{\text {reh }}$ parameters, and their prior distribution naturally stems from the underlying theoretical assumptions.

Instead, starting only with, say, $\epsilon_{1 *}=\beta\left(\Delta N_{*}\right)^{-\alpha}$, there is no guidance to choose the priors on $\alpha, \beta$ (for the inconsistencies in choosing $\Delta N_{*}$; see Sec. IV). In the absence of any other information, a simple guess would be, for instance, to take a flat prior on $\alpha$ and $\beta$. But if the purpose of $\epsilon_{1 *}(N)$ is to actually represent an inflationary model, then $\alpha=\alpha\left(\boldsymbol{\theta}_{\text {inf }}\right)$ and $\beta=\beta\left(\boldsymbol{\theta}_{\text {inf }}\right)$ such that flat priors on $\alpha$ and $\beta$ would correspond to unnatural priors on the $\boldsymbol{\theta}_{\text {inf }}$ and $\boldsymbol{\theta}_{\text {reh }}$ parameters. As a matter of fact, Bayesian evidence derived in such a way would be flawed.

Let us now illustrate the above considerations with a very simple model, loop inflation (LI), the potential of which is given by $V(\phi)=M^{4}\left[1+\alpha_{\mathrm{LI}} \ln \left(\phi / M_{\mathrm{P} 1}\right)\right]$. It is characterized by two parameters, the mass scale $M$ and $\alpha_{\mathrm{LI}}$. We therefore have $\boldsymbol{\theta}_{\text {inf }}=\left\{M, \alpha_{\mathrm{LI}}\right\}$. At leading order in slow roll, LI is a model like $\mathrm{SFI}_{2}$ for which the expansion in $1 / \Delta N_{*}$ does not enter any known classification. An expansion in $\alpha_{\mathrm{LI}}$ may, however, be consistently performed, and one gets

$$
\begin{aligned}
& \epsilon_{1 *}=\frac{\alpha_{\mathrm{LI}}}{\Delta N_{*}}+\mathcal{O}\left[\alpha_{\mathrm{LI}}^{2} \ln \left(\alpha_{\mathrm{LI}} \Delta N_{*}\right)\right], \\
& \epsilon_{2 *}=\frac{1}{\Delta N_{*}}+\mathcal{O}\left(\frac{\alpha_{\mathrm{LI}}}{\Delta N_{*}}\right) .
\end{aligned}
$$

This means that, for this model, $\alpha\left(\boldsymbol{\theta}_{\text {inf }}\right)=1$ and $\beta\left(\boldsymbol{\theta}_{\text {inf }}\right)=$ $\alpha_{\mathrm{LI}} / 4$. Ignoring the underlying model and just postulating $\epsilon_{1 *}=\beta\left(\Delta N_{*}\right)^{-\alpha}$, one would be tempted to choose a flat prior on $\beta$, i.e. a flat prior on $\alpha_{\mathrm{LI}}$. But within loop inflation, $\alpha_{\mathrm{LI}}$ is a coupling constant as the logarithm in the expression of the potential originates from a one-loop calculation. As a consequence, $\alpha_{\mathrm{LI}}$ is a small parameter, the order of magnitude of which is unknown a priori. Therefore, an uninformative prior for $\alpha_{\mathrm{LI}}$ is a Jeffreys' prior. Assuming a flat prior would not lead to equal probability per decade and would bias $\alpha_{\mathrm{LI}}$ toward unnatural large values.

It is then worth recalling that changing the prior may modify the posterior since

$$
P\left(\alpha_{\mathrm{LI}} \mid D\right)=\frac{1}{P(D)} \mathcal{L}\left(D \mid \alpha_{\mathrm{LI}}\right) \pi\left(\alpha_{\mathrm{LI}}\right)
$$

Depending on how peaked the likelihood $\mathcal{L}\left(D \mid \alpha_{\mathrm{LI}}\right)$ is, different $\pi\left(\alpha_{\mathrm{LI}}\right)$ would lead to different $P\left(\alpha_{\mathrm{LI}} \mid D\right)$. More importantly, an incorrect prior would also change the global likelihood $P(D)$, and thus the Bayesian evidence. As an illustration, let us consider a toy likelihood function which is a simple Gaussian

$$
\mathcal{L}=\mathcal{L}_{\max } e^{-\alpha_{\mathrm{LI}}^{2} /\left(2 \sigma^{2}\right)}
$$

then for a flat prior $\pi^{\mathrm{b}}\left(\alpha_{\mathrm{LI}}\right)=1 / \Delta \alpha_{\mathrm{LI}}$ with $\Delta \alpha_{\mathrm{LI}} \equiv$ $\alpha_{\mathrm{LI}}^{\max }-\alpha_{\mathrm{LI}}^{\min }$, one obtains

$P^{b}(D)=\mathcal{L}_{\max } \sqrt{\frac{\pi}{2}} \frac{\sigma}{\Delta \alpha_{\mathrm{LI}}}\left[\operatorname{erf}\left(\frac{\alpha_{\mathrm{LI}}^{\min }}{\sigma \sqrt{2}}\right)-\operatorname{erf}\left(\frac{\alpha_{\mathrm{LI}}^{\max }}{\sigma \sqrt{2}}\right)\right]$,

where $\operatorname{erf}(x)$ is the error function. For a Jeffreys' prior $\pi^{\natural}\left(\alpha_{\mathrm{LI}}\right)=1 /\left[\alpha_{\mathrm{LI}} \ln \left(\alpha_{\mathrm{LI}}^{\max } / \alpha_{\mathrm{LI}}^{\min }\right)\right]$, one has

$$
\begin{aligned}
P^{\natural}(D)= & \frac{\mathcal{L}_{\max }}{2 \ln \left(\alpha_{\mathrm{LI}}^{\max } / \alpha_{\mathrm{LI}}^{\min }\right)} \\
& \times\left\{E_{1}\left[\frac{\left(\alpha_{\mathrm{LI}}^{\min }\right)^{2}}{2 \sigma^{2}}\right]-E_{1}\left[\frac{\left(\alpha_{\mathrm{LI}}^{\max }\right)^{2}}{2 \sigma^{2}}\right]\right\},
\end{aligned}
$$

where $E_{1}(z)=\int_{z}^{\infty} \mathrm{d} t e^{-t} / t$ is an exponential integral function. Viewed as functions of $\alpha_{\mathrm{LI}}^{\min }$ and $\alpha_{\mathrm{LI}}^{\max }$, the previous "toy model calculation" illustrates the fact that the Bayesian evidence can be very different according to assumptions made on the prior distributions for the $\boldsymbol{\theta}_{\text {inf }}$ 's.

We therefore conclude that considering $\epsilon_{1 *}=\beta\left(\Delta N_{*}\right)^{-\alpha}$ without reference to an underlying theoretical framework leads to uninformative statistical results. If one ignores the fact that $\alpha=\alpha\left(\boldsymbol{\theta}_{\text {inf }}\right)$ and $\beta=\beta\left(\boldsymbol{\theta}_{\text {inf }}\right)$, our ability to fix different priors for different models is lost. As a consequence, this approach is not well suited to carry out model comparison and derive statistical constraints on the physics of the early Universe.

\section{CONCLUSIONS}

In this short article, we have argued that it is often too simplistic to view inflation as a framework that can be "described by two numbers." The goal of a model is not to predict the values of $n_{\mathrm{s}}$ and $r$ only. In fact, it should first predict the amplitude of the cosmological perturbations, as some models actually do (SI, CWI, OSTI, DI). Then, even if inflation is featureless, single field, and slowly rolling, with minimal kinetic terms, one can still reasonably hope to measure other numbers, such as the running $\alpha_{\mathrm{s}}$. But more importantly, inflation does not only consist in a phase of accelerated expansion. The mechanism that ends inflation is also of crucial importance and, as a matter of fact, can be constrained by CMB data $[82,84,87]$. The new parametrizations miss this opportunity. They can never be as informative as an approach rooted in field theory, or some specific modified gravity framework $[115,116]$, when it comes to a phenomenon that could have taken place at an energy scale as high as $10^{16} \mathrm{GeV}$ [117]. At last, specifying a model in the hope of comparing it with some data also 
means giving the priors on its free parameters to ensure its internal consistency. This is usually much more than specifying two numbers.

The price to pay is that some predictions do depend on the underlying model, but not all of them. For instance, a generic prediction of inflation is the presence of Doppler peaks in the CMB which makes inflation a falsifiable scenario. On the other hand, there is no generic prediction for $r$, except that it must be such that the energy scale of inflation is higher than the one of $\mathrm{BBN}$, leading to a ridiculously small lower bound, $r \gtrsim 10^{-75}$, a value which is unobservable as smaller than backreaction effects [118]. But this does not necessarily mean that the situation is not interesting; models do predict different ranges of tensor-toscalar ratio values, and measuring $r$ provides information about the underlying inflationary scenario.

One of the goals of phenomenological parametrizations was to narrow down these ranges and yield "typical" inflationary predictions. For instance, it is often argued that, while $\epsilon_{1 *}=\mathcal{O}(1) / \Delta N_{*}$ (yielding $r \simeq 0.26$ for $\left.\Delta N_{*}=60\right)$ is now excluded by the data, the next target according to Eq. (13) would be to try and detect the next order in $1 / \Delta N_{*}$, namely, $\epsilon_{1 *}=\mathcal{O}(1) / \Delta N_{*}^{2}$ (yielding $r \simeq 0.004$ for $\Delta N_{*}=60$ ). However, nothing guarantees that the overall constant is indeed of order 1 . For instance, as can be seen in Eq. (48), this is the case for the model LI since $\alpha_{\mathrm{LI}} \ll 1$. In fact, a value less than 0.25 is already sufficient to reestablish the agreement between the prediction $\epsilon_{1 *}=\mathcal{O}(1) / \Delta N_{*}$ and the data.

In conclusion, it seems to us that, even if the phenomenological parametrizations discussed in the present work may provide useful rule-of thumb classifications, the most promising method to learn about the physics of inflation is to build models based on high energy physics and (modified) gravity, since this is a priori the way nature has realized inflation in practice. At the time when the Planck data tell us that the Higgs field of particle physics, some low energy String compactifications, or the $R^{2}$ corrections to General Relativity [20] could explain the large scale structure of the Universe, it seems that phenomenological parametrizations are not sufficient to tackle the physical questions we now have to address. The fact that some predictions are model dependent is not a shortcoming but actually a virtue of inflation since it can be used to learn about physics in a regime hardly achievable with current technology.

\section{ACKNOWLEDGMENTS}

It is a pleasure to thank Diederik Roest for interesting discussions and useful comments. V. V.'s work is supported by STFC Grants No. ST/K00090X/1 and No. ST/L005573/ 1. C. R. is partially supported by the Belgian Federal Office for Science, Technical \& Cultural Affairs through the Interuniversity Attraction Pole P7/37.
[1] A. A. Starobinsky, JETP Lett. 30, 682 (1979) [Pisma Zh. Eksp. Teor. Fiz. 30, 719 (1979)].

[2] A. A. Starobinsky, Phys. Lett. 91B, 99 (1980).

[3] A. H. Guth, Phys. Rev. D 23, 347 (1981).

[4] A. D. Linde, Phys. Lett. 108B, 389 (1982).

[5] A. Albrecht and P. J. Steinhardt, Phys. Rev. Lett. 48, 1220 (1982).

[6] A. D. Linde, Phys. Lett. 129B, 177 (1983).

[7] V. F. Mukhanov and G. V. Chibisov, JETP Lett. 33, 532 (1981) [Pis'ma Zh. Eksp. Teor. Fiz. 33, 549 (1981)].

[8] V. F. Mukhanov and G. V. Chibisov, Sov. Phys. JETP 56, 258 (1982) [Zh. Eksp. Teor. Fiz. 83, 475 (1982)].

[9] A. A. Starobinsky, Phys. Lett. 117B, 175 (1982).

[10] A. H. Guth and S. Y. Pi, Phys. Rev. Lett. 49, 1110 (1982).

[11] S. W. Hawking, Phys. Lett. 115B, 295 (1982).

[12] J. M. Bardeen, P. J. Steinhardt, and M. S. Turner, Phys. Rev. D 28, 679 (1983).

[13] P. Ade et al. (Planck Collaboration), Astron. Astrophys. 571, A1 (2014).

[14] R. Adam et al. (Planck Collaboration), Astron. Astrophys. 594, A1 (2016).

[15] P. A. R. Ade et al. (Planck Collaboration), Astron. Astrophys. 594, A13 (2016).
[16] P. A. R. Ade et al. (Planck Collaboration), Astron. Astrophys. 594, A20 (2016).

[17] J. Martin, C. Ringeval, and R. Trotta, Phys. Rev. D 83, 063524 (2011).

[18] R. Easther and H. V. Peiris, Phys. Rev. D 85, 103533 (2012).

[19] J. Martin, C. Ringeval, and V. Vennin, Phys. Dark Univ. 5-6, 75 (2014).

[20] J. Martin, C. Ringeval, R. Trotta, and V. Vennin, J. Cosmol. Astropart. Phys. 03 (2014) 039.

[21] L. C. Price, H. V. Peiris, J. Frazer, and R. Easther, J. Cosmol. Astropart. Phys. 02 (2016) 049.

[22] J. Martin, Astrophys. Space Sci. Proc. 45, 41 (2016).

[23] X. Chen and C. Ringeval, J. Cosmol. Astropart. Phys. 08 (2012) 014.

[24] V. Vennin, K. Koyama, and D. Wands, J. Cosmol. Astropart. Phys. 11 (2015) 008.

[25] V. Vennin, K. Koyama, and D. Wands, J. Cosmol. Astropart. Phys. 03 (2016) 024.

[26] X. Chen, C. Dvorkin, Z. Huang, M. H. Namjoo, and L. Verde, J. Cosmol. Astropart. Phys. 11 (2016) 014.

[27] V. F. Mukhanov, JETP Lett. 41, 493 (1985) [Pisma Zh. Eksp. Teor. Fiz. 41, 402 (1985)]. 
[28] V. F. Mukhanov, Sov. Phys. JETP 67, 1297 (1988) [Zh. Eksp. Teor. Fiz. 94N7, 1 (1988)].

[29] E. D. Stewart and D. H. Lyth, Phys. Lett. B 302, 171 (1993).

[30] J.-O. Gong and E. D. Stewart, Phys. Lett. B 510, 1 (2001).

[31] D. J. Schwarz, C. A. Terrero-Escalante, and A. A. Garcia, Phys. Lett. B 517, 243 (2001).

[32] S. M. Leach, A. R. Liddle, J. Martin, and D. J. Schwarz, Phys. Rev. D 66, 023515 (2002).

[33] W. H. Kinney and K. Tzirakis, Phys. Rev. D 77, 103517 (2008).

[34] K. Tzirakis and W. H. Kinney, J. Cosmol. Astropart. Phys. 01 (2009) 028.

[35] L. Lorenz, J. Martin, and C. Ringeval, Phys. Rev. D 78, 083513 (2008).

[36] N. Agarwal and R. Bean, Phys. Rev. D 79, 023503 (2009).

[37] J. Martin, C. Ringeval, and V. Vennin, J. Cosmol. Astropart. Phys. 06 (2013) 021.

[38] J. Beltran Jimenez, M. Musso, and C. Ringeval, Phys. Rev. D 88, 043524 (2013).

[39] T. T. Nakamura and E. D. Stewart, Phys. Lett. B 381, 413 (1996).

[40] R. Easther and J. T. Giblin, Phys. Rev. D 72, 103505 (2005).

[41] F. Di Marco and F. Finelli, Phys. Rev. D 71, 123502 (2005).

[42] T. Battefeld and R. Easther, J. Cosmol. Astropart. Phys. 03 (2007) 020.

[43] T. Chiba and M. Yamaguchi, J. Cosmol. Astropart. Phys. 01 (2009) 019.

[44] A. De Felice and S. Tsujikawa, J. Cosmol. Astropart. Phys. 02 (2012) 007.

[45] X. Chen, M.-x. Huang, S. Kachru, and G. Shiu, J. Cosmol. Astropart. Phys. 01 (2007) 002.

[46] S. Yokoyama, T. Suyama, and T. Tanaka, J. Cosmol. Astropart. Phys. 07 (2007) 013.

[47] K. Ichikawa, T. Suyama, T. Takahashi, and M. Yamaguchi, Phys. Rev. D 78, 023513 (2008).

[48] D. Langlois, S. Renaux-Petel, D. A. Steer, and T. Tanaka, Phys. Rev. D 78, 063523 (2008).

[49] X. Chen, Adv. Astron. 2010, 1 (2010).

[50] D. J. Schwarz and C. A. Terrero-Escalante, J. Cosmol. Astropart. Phys. 08 (2004) 003.

[51] P. Creminelli and M. Zaldarriaga, J. Cosmol. Astropart. Phys. 10 (2004) 006.

[52] C. Cheung, P. Creminelli, A. L. Fitzpatrick, J. Kaplan, and L. Senatore, J. Cosmol. Astropart. Phys. 03 (2008) 014.

[53] L. Amendola et al., arXiv:1606.00180.

[54] M. B. Hoffman and M. S. Turner, Phys. Rev. D 64, 023506 (2001).

[55] W. H. Kinney, Phys. Rev. D 66, 083508 (2002).

[56] E. Ramirez and A. R. Liddle, Phys. Rev. D 71, 123510 (2005).

[57] S. Chongchitnan and G. Efstathiou, Phys. Rev. D 72, 083520 (2005).

[58] Q.-G. Huang, Phys. Rev. D 76, 061303 (2007).

[59] D. Roest, J. Cosmol. Astropart. Phys. 01 (2014) 007.
[60] J. Garcia-Bellido and D. Roest, Phys. Rev. D 89, 103527 (2014).

[61] P. Binetruy, E. Kiritsis, J. Mabillard, M. Pieroni, and C. Rosset, J. Cosmol. Astropart. Phys. 04 (2015) 033.

[62] Q.-G. Huang, K. Wang, and S. Wang, Phys. Rev. D 93, 103516 (2016).

[63] P. Creminelli, S. Dubovsky, D. López Nacir, M. Simonović, G. Trevisan, G. Villadoro, and M. Zaldarriaga, Phys. Rev. D 92, 123528 (2015).

[64] V. Mukhanov, Eur. Phys. J. C 73, 2486 (2013).

[65] A. R. Liddle, Phys. Rev. D 68, 103504 (2003).

[66] V. Vennin, Phys. Rev. D 89, 083526 (2014).

[67] D. Coone, D. Roest, and V. Vennin, J. Cosmol. Astropart. Phys. 11 (2015) 010.

[68] X. Chen, H. Firouzjahi, M. H. Namjoo, and M. Sasaki, J. Cosmol. Astropart. Phys. 09 (2013) 012.

[69] D. S. Salopek, J. R. Bond, and J. M. Bardeen, Phys. Rev. D 40, 1753 (1989).

[70] I. J. Grivell and A. R. Liddle, Phys. Rev. D 61, 081301 (2000).

[71] J. A. Adams, B. Cresswell, and R. Easther, Phys. Rev. D 64, 123514 (2001).

[72] S. Tsujikawa, D. Parkinson, and B. A. Bassett, Phys. Rev. D 67, 083516 (2003).

[73] D. Parkinson, S. Tsujikawa, B. A. Bassett, and L. Amendola, Phys. Rev. D 71, 063524 (2005).

[74] A. Makarov, Phys. Rev. D 72, 083517 (2005).

[75] C. Ringeval, Lect. Notes Phys. 738, 243 (2008).

[76] A. R. Liddle, P. Parsons, and J. D. Barrow, Phys. Rev. D 50, 7222 (1994).

[77] T. Matsumura et al., J. Low Temp. Phys. 176, 733 (2014).

[78] COrE, A satellite mission for probing cosmic origins, neutrinos masses and the origin of stars and magnetic fields, http://www.core-mission.org/science.php.

[79] J. Beltran Jimenez, L. Heisenberg, G. J. Olmo, and C. Ringeval, J. Cosmol. Astropart. Phys. 11 (2015) 046.

[80] A. R. Liddle and S. M. Leach, Phys. Rev. D 68, 103503 (2003).

[81] J. Martin and C. Ringeval, J. Cosmol. Astropart. Phys. 08 (2006) 009.

[82] J. Martin and C. Ringeval, Phys. Rev. D 82, 023511 (2010).

[83] C. Ringeval, T. Suyama, and J. Yokoyama, J. Cosmol. Astropart. Phys. 09 (2013) 020.

[84] J. Martin, C. Ringeval, and V. Vennin, Phys. Rev. Lett. 114, 081303 (2015).

[85] L. Dai, M. Kamionkowski, and J. Wang, Phys. Rev. Lett. 113, 041302 (2014).

[86] M. Drewes, J. Cosmol. Astropart. Phys. 03 (2016) 013.

[87] J. Martin, C. Ringeval, and V. Vennin, Phys. Rev. D 93, 103532 (2016).

[88] T. Terada, Y. Watanabe, Y. Yamada, and J. Yokoyama, J. High Energy Phys. 02 (2015) 105.

[89] F. Bezrukov and M. Shaposhnikov, Phys. Lett. B 659, 703 (2008).

[90] J. Garcia-Bellido, D. G. Figueroa, and J. Rubio, Phys. Rev. D 79, 063531 (2009).

[91] D. G. Figueroa, J. Garcia-Bellido, and F. Torrenti, Phys. Rev. D 92, 083511 (2015). 
[92] J. Repond and J. Rubio, J. Cosmol. Astropart. Phys. 07 (2016) 043.

[93] J. Martin, C. Ringeval, and V. Vennin, J. Cosmol. Astropart. Phys. 10 (2014) 038.

[94] J. D. Barrow and P. Saich, Phys. Lett. B 249, 406 (1990).

[95] J. D. Barrow and A. R. Liddle, Phys. Rev. D 47, R5219 (1993).

[96] P. Parsons and J. D. Barrow, Phys. Rev. D 51, 6757 (1995).

[97] J. D. Barrow and N. J. Nunes, Phys. Rev. D 76, 043501 (2007).

[98] J. Martin, C. Ringeval, and V. Vennin, Phys. Dark Univ. 5, 75 (2014).

[99] L. Abbott, Nucl. Phys. B185, 233 (1981).

[100] J. R. Ellis, D. V. Nanopoulos, K. A. Olive, and K. Tamvakis, Nucl. Phys. B221, 524 (1983).

[101] A. Albrecht, S. Dimopoulos, W. Fischler, E. W. Kolb, S. Raby, and P. J. Steinhardt, Nucl. Phys. B229, 528 (1983).

[102] E. Witten, Phys. Rev. D 46, 5467 (1992).

[103] E. Witten, Phys. Rev. D 47, 3405 (1993).

[104] A. A. Gerasimov and S. L. Shatashvili, J. High Energy Phys. 10 (2000) 034.

[105] D. Kutasov, M. Marino, and G. W. Moore, J. High Energy Phys. 10 (2000) 045.
[106] L. Kofman and A. D. Linde, J. High Energy Phys. 07 (2002) 004.

[107] L. Alvarez-Gaume, J. Distler, C. Kounnas, and M. Marino, Int. J. Mod. Phys. A 11, 4745 (1996).

[108] J. Garcia-Bellido, Phys. Lett. B 418, 252 (1998).

[109] K. A. Malik and D. Wands, J. Cosmol. Astropart. Phys. 02 (2005) 007.

[110] V. F. Mukhanov, H. A. Feldman, and R. H. Brandenberger, Phys. Rep. 215, 203 (1992).

[111] C. Ringeval, Mon. Not. R. Astron. Soc. 439, 3253 (2014).

[112] R. Trotta, F. Feroz, M. P. Hobson, L. Roszkowski, and R. Ruiz de Austri, J. High Energy Phys. 12 (2008) 024.

[113] R. Trotta, Contemp. Phys. 49, 71 (2008).

[114] J. Martin, C. Ringeval, R. Trotta, and V. Vennin, Phys. Rev. D 90, 063501 (2014).

[115] A. De Felice and S. Tsujikawa, Living Rev. Relativ. 13, 3 (2010).

[116] A. De Felice and S. Tsujikawa, Phys. Rev. D 84, 083504 (2011).

[117] F. Bezrukov, J. Rubio, and M. Shaposhnikov, Phys. Rev. D 92, 083512 (2015).

[118] P. Martineau and R. Brandenberger, Mod. Phys. Lett. A 23, 727 (2008). 